\title{
Differential expression of extracellular matrix-related genes DCN, EPHA4, FN1, SPARC, SPON2 and SPP1 in colorectal carcinogenesis
}

\author{
MARGARETA ŽLAJPAH, NINA HAUPTMAN, EMANUELA BOŠTJANČIČ and NINA ZIDAR
}

Institute of Pathology, Faculty of Medicine, University of Ljubljana, 1000 Ljubljana, Slovenia

Received February 20, 2019; Accepted July 17, 2019

DOI: 10.3892/or.2019.7274

\begin{abstract}
During colorectal carcinogenesis, a spectrum of lesions is formed with different malignant potentials and occasionally with ambiguous morphologic features. To identify novel biomarkers, we previously performed a bioinformatics study to detect differentially expressed genes between colorectal adenoma (CRA) and colorectal carcinoma (CRC). The present study validated the diagnostic and prognostic significance of 6 components of the extracellular matrix (ECM) that were identified in the previous bioinformatics analysis [decorin (DCN), erythropoietin-producing hepatoma receptor A4 (EPHA4), fibronectin 1 (FN1), secreted protein acidic and cysteine rich (SPARC), spondin 2 (SPON2) and secreted phosphoprotein 1 (SPP1)], by analyzing their gene and protein expression levels in all samples. A total of 60 formalin-fixed paraffin-embedded biopsy samples were included in the present study, from 40 patients with CRA, malignant polyps and CRC with and without lymph node metastases. The expression of the ECM-related genes was evaluated on the mRNA level using reverse transcription-quantitative PCR (RT-qPCR) and on the protein level using immunohistochemistry. RT-qPCR results revealed differential expression among the groups for all genes, except for EPHA4. Their expression proportionally increased with the level of malignancy. Among them, SPARC, SPON2 and SPP1 were differentially expressed between CRA and malignant polyps. Immunohistochemistry analysis revealed two patterns: Positive staining of SPON2 and SPP1 in epithelial cells of healthy mucosa and dysplastic glands of CRA and CRC, and positive staining of DCN and SPARC in the lamina propria in healthy mucosa and stroma of CRA and CRC. The intensity of staining increased with the severity of lesions. The present results suggested that ECM-related proteins may have an important role in the development of
\end{abstract}

Correspondence to: Dr Nina Zidar, Institute of Pathology, Faculty of Medicine, University of Ljubljana, Korytkova 2, 1000 Ljubljana, Slovenia

E-mail: nina.zidar@mf.uni-lj.si

Key words: colorectal carcinoma, colorectal adenoma, extracellular matrix, quantitative real-time PCR, immunohistochemistry
CRC, with a possible diagnostic use for differentiation among various lesions, such as between CRA and malignant polyps. However, no significant potential was detected for these genes to predict lymph node metastases in CRC.

\section{Introduction}

Colorectal carcinoma (CRC) arises as a result of genetic and epigenetic changes in epithelial cells (1-3). Upon its development, a spectrum of lesions are formed, from colorectal adenoma (CRA) to $\mathrm{CRC}$, with different malignant potential and behavior (3). Since the introduction of colon cancer screening programs, a large number of early lesions have been discovered and removed, including adenomas with epithelial misplacement (pseudoinvasion) and malignant polyps (early carcinomas) (4-6). Despite well-defined morphological features of adenomas with epithelial misplacement and malignant polyps (7), there is a significant number of problematic cases of precancerous lesions with ambiguous features, leading to different diagnostic opinions among pathologists (5). Biomarkers distinguishing among various lesions in colorectal carcinogenesis could therefore help to make the correct diagnosis in ambiguous cases, thus enabling the optimal treatment to be selected $(8,9)$.

In a previous study from our group, the most prominent gene candidates were examined in order to distinguish CRA from CRC using bioinformatics analysis (9). Using a public genomics data repository (Gene Expression Omnibus), several projects were identified with gene expression profiles of normal, adenoma and carcinoma colonic samples, obtained from microarray experiments. Applying in silico methods, 16 differentially expressed genes were detected between CRA and CRC and 10 differentially expressed genes were detected among healthy colon mucosa, CRA and CRC. Functional analysis of these differentially expressed genes indicated that at least 9 of them were associated with the extracellular matrix (ECM).

The ECM is assembled from diverse biochemical molecules ranging from proteins to polysaccharides $(10,11)$, including proteoglycans and glycoproteins, with unique physical and biochemical properties. The ECM offers structural support to organs (12) and participates in crucial cell processes, including proliferation, differentiation, adhesion, migration and apoptosis (10). Notably, the ECM also participates in many cellular functions representing the hallmarks of cancer. This overlap 
suggests that the biochemical and biophysical properties of ECM should be considered when examining tumour behaviour and therapeutic interventions (13).

Only 3 out of the 9 genes that were differentially expressed between healthy colon mucosa, CRA and CRC, encode for collagens, namely collagen type XII $\alpha 1$ chain, collagen type II $\alpha 1$ chain and collagen type III $\alpha 1$ chain. The remaining 6 genes [decorin (DCN), erythropoietin-producing hepatoma receptor A4 (EPHA4), fibronectin 1 (FN1), secreted protein acidic and cysteine rich (SPARC), spondin 2 (SPON2) and secreted phosphoprotein 1 (SPP1)], have enzymatic activities. Structural proteins, such as these three collagens, are known to participate in biomechanical signaling, but do not have an enzymatic role in tumour progression (14). Changes in collagen expression and ratio enable tumour progression, possible due to alterations in the enzymatic activity of ECM proteins, such as DCN, EPH4, FN1, SPARC, SPON2 and SPP1. The aim of the present study was to examine the diagnostic and prognostic potential of these 6 ECM-related components with enzymatic activity in colorectal carcinogenesis. All 6 genes were identified as being differentially expressed in our previous bioinformatics analysis comparing CRA and CRC (9). All of these 6 components of ECM have been already associated with CRC (15-38). However, there are limited data about how the expression levels of mRNAs of these genes and their related proteins change from healthy mucosa and CRA to malignant polyps and CRC, including the development of metastases. The few published studies have mainly focused on either protein and/or mRNAs levels (18-22). On the basis of the literature and our previous bioinformatics analysis (9), it was hypothesized that the expression of these 6 ECM-related genes may be different, on both mRNA and protein levels, in healthy mucosa and CRA in comparison to malignant polyps and CRC (diagnostic potential), as well as between CRC with and without lymph node metastases (prognostic potential).

\section{Materials and methods}

Patients and tissue selection. The present study included biopsy samples from 40 patients divided into four groups on the basis of the biopsy diagnosis: CRA, malignant polyp, CRC without lymph node metastases (CRC N0), or CRC with lymph node metastases (CRC N+). The gender and age of the patients, and the location of the lesions for each group, are listed in Table I. Only cases with clear biopsy diagnoses were included. In the CRA group, there were 4 tubular adenomas with high grade dysplasia, 4 tubulovillous adenomas with high grade dysplasia and 2 tubulovillous adenomas with low grade dysplasia. The malignant polyps group consisted of 5 tubulovillous adenomas, 3 tubular adenomas, 1 villous adenoma and 1 tubular adenoma, all with high grade dysplasia and with malignant transformation, evidenced by invasion of the dysplastic glands in the submucosa (pT1 stage). The TNM stages of the CRC N0 group were T2NOM0, T3N0M0 and T4aNOM0 in 2, 6 and 2 patients, respectively. The TNM stages of the CRC N+ group were T3N1Mx, T4aN1Mx and T4aN2Mx in 5, 2 and 3 patients, respectively.

As a control group, healthy colon mucosa from surgical margins from 20 patients with CRC N0 and CRC N+ was included.
All tissue samples were fixed for $24 \mathrm{~h}$ in $10 \%$ buffered formalin prior to the paraffin embedding. After fixation and embedding, tissues were cut into 3-4 $\mu \mathrm{m}$ sections and stained with hematoxylin and eosin for routine histopathological examination. For the purposes of the present retrospective study, representative paraffin blocks were retrieved from the archives of the Institute of Pathology, Faculty of Medicine, University of Ljubljana (tissues originally collected from December 2013 to October 2018).

\section{Isolation of RNA from formalin-fixed paraffin-embedded (FFPE) tissue}

Isolation from tissue sections. Four $10 \mu \mathrm{m}$-thick FFPE sections were cut from the archival paraffin blocks for the isolation of total RNA. Total RNA extraction was performed using the AllPrep DNA/RNA FFPE kit (Qiagen GmbH), according to the manufacturer's instructions. Apart from the deparaffinization solution (hexadecane; Sigma-Aldrich; Merck KGaA) and the ethanol (Merck KGaA), all the reagents were from Qiagen $\mathrm{GmbH}$. The RNA concentration and quality was evaluated using a NanoDrop 1000 spectrophotometer (Thermo Fisher Scientific, Inc.). The A260/A230 nm intensity ratio was $>1.0$ and the A260/ A280 ratio was $>1.8$. Reverse transcription and amplification using SYBR Green technology of the housekeeping gene GAPDH (95 bp) was performed as quality control. Reverse transcription was performed as described below. Each qPCR reaction contained $1 \mu 1$ 10X GAPDH primers (QuantiTect primer assay Hs_GAPDH_1_S; cat. no. QT00079247; Qiagen GmbH), $5 \mu \mathrm{l}$ iTaq Universal SYBR Green (Bio-Rad Laboratories, Inc.) and $4 \mu \mathrm{l}$ of cDNA. Thermocycling conditions were as follows: $2 \mathrm{~min}$ at $50^{\circ} \mathrm{C}, 2 \mathrm{~min}$ at $95^{\circ} \mathrm{C}$, and 45 cycles of $15 \mathrm{sec}$ at $94^{\circ} \mathrm{C}$, $30 \mathrm{sec}$ at $55^{\circ} \mathrm{C}$ and $1 \mathrm{~min}$ at $72^{\circ} \mathrm{C}$. This approach is similar to the approach that we use for FusionPlex Sarcoma, a next-generation sequencing panel, which includes the PreSeq RNA QC assay (39) after reverse transcription. These quality control assays basically use primers that amplify a universally expressed transcript (in the present study, GAPDH) to quantify the amount of amplifiable RNA of sufficient length in a sample. The presence of lower Cqs in the results is strongly indicative of successful cDNA preparation and predicts successful sequencing. All of the samples that were included in the present study passed amplification of GAPDH using SYBR Green (initial quality control) and those that did not amplify were not included in the study (the number of samples initially isolated was $>2$-fold the number of samples that passed quality control and were subsequently included in the final manuscript). Finally, for the selected genes, TaqMan probes (Thermo Fisher Scientific, Inc.) were designed to amplify and detect PCR products $<84$ bp long (Table SI).

Isolation from tissue cores. Tissue cores from the tumor center and the invasive front were punched from FFPE tissue blocks using a $600 \mu \mathrm{m}$ needle. Three punches were used for each isolation. Total RNA extraction was performed using the MagMax FFPE DNA/RNA Ultra kit (Applied Biosystems; Thermo Fisher Scientific, Inc.), according to the manufacturer's instructions, with one modification: Protease digestion was performed overnight with mixing for $15 \mathrm{sec}$ at $300 \mathrm{rpm}$ every $4 \mathrm{~min}$ instead of $1 \mathrm{~h}$. Apart from the deparaffinization solution (xylene; Sigma-Aldrich; Merck KGaA) and the ethanol (Merck KGaA), all the reagents were from Applied 
Table I. Summary of clinical data including location of the lesions.

\begin{tabular}{|c|c|c|c|c|}
\hline & Colorectal adenoma & Malignant polyp & CRC NO & $\mathrm{CRCN}+$ \\
\hline Number of patients & 10 & 10 & 10 & 10 \\
\hline Male : female & 10:0 & $6: 4$ & $3: 7$ & $7: 4$ \\
\hline Age & $50-83$ & $56-72$ & $48-84$ & $58-90$ \\
\hline$($ mean $\pm \mathrm{SD})$ & $(65 \pm 11)$ & $(65 \pm 5)$ & $(73 \pm 12)$ & $(77 \pm 11)$ \\
\hline \multicolumn{5}{|l|}{ Tumour location } \\
\hline Ascending colon & 1 & 0 & 1 & 1 \\
\hline Descending colon & 0 & 1 & 0 & 1 \\
\hline Right hemicolon & 0 & 0 & 2 & 2 \\
\hline Rectum or rectosigmoid colon & 2 & 3 & 2 & 4 \\
\hline Sigmoid colon & 6 & 6 & 4 & 1 \\
\hline Splenic flexure & 1 & 0 & 1 & 1 \\
\hline
\end{tabular}

CRC, colorectal carcinoma; N0, no lymph node metastases; N+, with lymph node metastases.

Biosystems (Thermo Fisher Scientific, Inc). RNA quantity was assessed on a NanoDrop 1000 (Thermo Fisher Scientific, Inc.) by measuring the absorbance at A 260 .

Reverse transcription-quantitative PCR (RT-qPCR). Reverse transcription, preamplification and $\mathrm{qPCR}$ were performed to measure the expression levels of genes. The extracted total RNA was reverse transcribed using the OneTaq-PCR kit (New England Biolabs, Inc.) according to manufacturer's instructions. Each reverse transcription reaction contained $60 \mathrm{ng}$ of extracted total RNA, $6 \mu \mathrm{M}$ random primers mix, $5 \mu 12 \mathrm{X}$ reaction mix and $1 \mu \mathrm{l} 10 \mathrm{X}$ enzyme mix. First, RNA and random primers were mixed and incubated for $5 \mathrm{~min}$ at $70^{\circ} \mathrm{C}$, then the reaction and enzyme mix was added and incubated for $5 \mathrm{~min}$ at $25^{\circ} \mathrm{C}, 60 \mathrm{~min}$ at $42^{\circ} \mathrm{C}$ and $4 \mathrm{~min}$ at $80^{\circ} \mathrm{C}$.

Following cDNA synthesis, a preamplification procedure was performed using TaqMan PreAmp mastermix (Applied Biosystems; Thermo Fisher Scientific, Inc.). Each reaction contained $5 \mu \mathrm{l}$ cDNA, $5 \mu \mathrm{l}$ of $0.2 \mathrm{X}$ TaqMan Gene Expression Assays (Table SI) and $10 \mu 1$ 2X TaqMan PreAmp Mastermix. TaqMan Gene Expression Assays were pooled, followed by dilution to $0.2 \mathrm{X}$ using Tris-EDTA buffer solution, $\mathrm{pH} 8.0$ (Sigma-Aldrich; Merck KGaA). The thermocycling conditions were as follows: $10 \mathrm{~min}$ at $95^{\circ} \mathrm{C}$ and 10 cycles of $15 \mathrm{sec}$ at $95^{\circ} \mathrm{C}$ and $4 \mathrm{~min}$ at $60^{\circ} \mathrm{C}$.

Prior to qPCR, to calculate the efficiency of qPCR reactions, pools of each group were created by mixing RNA samples. RNA pools were reverse transcribed and preamplified, as aforementioned. Then, the preamplified cDNA was diluted in four steps, ranging from 5-fold dilution to 5,000-fold or 625-fold and qPCR was performed as described below. All qPCR reactions were conducted on a Rotor-Gene Q system (Qiagen $\mathrm{GmbH}$ ), and each sample was run in triplicate. The thermocycling conditions were as follows: $2 \mathrm{~min}$ at $50^{\circ} \mathrm{C}, 10 \mathrm{~min}$ at $95^{\circ} \mathrm{C}$, and 40 cycles of $15 \mathrm{sec}$ at $95^{\circ} \mathrm{C}$ and $1 \mathrm{~min}$ at $60^{\circ} \mathrm{C}$.

qPCR was performed using TaqMan technology with FastStart Essential DNA Probe Master (Roche Diagnostics). Each preamplification reaction $(20 \mu \mathrm{l})$ was diluted with $\mathrm{ddH}_{2} \mathrm{O}$ to a final volume $100 \mu \mathrm{l}(5 \mathrm{X})$. Each qPCR reaction contained $4.5 \mu \mathrm{l}$ of diluted preamplification mixture, $5 \mu \mathrm{l}$ of $2 \mathrm{X}$ FastStart Essential DNA Probe Master (Roche Diagnostics) and $0.5 \mu \mathrm{l}$ of TaqMan Gene Expression Assay. The TaqMan Gene Expression assays used in the present study are listed in Table SI. Importin 8 (IPO8) and $\beta 2$ microglobulin (B2M) were used as reference genes. All qPCR reactions were conducted on a Rotor-Gene Q system (Qiagen $\mathrm{GmbH}$ ), and each sample was run in duplicate. The thermocycling conditions were as follows: $2 \mathrm{~min}$ at $50^{\circ} \mathrm{C}$, $10 \mathrm{~min}$ at $95^{\circ} \mathrm{C}$ and 40 cycles of $15 \mathrm{sec}$ at $95^{\circ} \mathrm{C}$ and $1 \mathrm{~min}$ at $60^{\circ} \mathrm{C}$.

Immunohistochemistry. For immunohistochemistry analysis, commercially available antibodies against SPP1, SPARC, SPON2 and DCN were used. Detailed information about the antibodies is presented in Table II. Sections of $4 \mu \mathrm{m}$ thickness were cut from paraffin blocks, deparaffinized, and processed for automated antigen retrieval and staining with the antibodies in an automatic immunostainer (Ventana BenchMark; Roche Diagnostics). Sections were treated with biotinylated secondary antibody $\left(8 \mathrm{~min}\right.$ at $37^{\circ} \mathrm{C}$ ), followed by incubation with peroxidase-conjugated streptavidin (both part of the UltraVIEW DAB Detection kit; Roche Diagnostics). Visualization of the immunoreaction was established with 3,3'-diaminobenzidine. The sections were then counterstained with haematoxylin.

Statistical analysis. To calculate relative gene expression, $\mathrm{Cq}$ values were corrected according to Latham (40). To obtain $\Delta \mathrm{Cq}$, the geometric mean of $\mathrm{Cq}$ values of the reference genes (IPO8 and B2M) was deducted from the gene of interest (DCN, EPH4, FN1, SPARC, SPON2 and SPP1). Differences in expression in the paired tissue samples of tumour and adjacent healthy mucosa from the CRC N0 group were analyzed for significance with the Wilcoxon signed rank test. For all other comparisons of $\Delta \mathrm{Cqs}$ between different groups of samples (healthy mucosa, CRA, malignant polyp, CRC N0 and $\mathrm{CRC} \mathrm{N}+$ ), ANOVA was used with two different post-hoc tests, Bonferroni and Hochberg's GT2 (41). Additionally, the Spearman's Rank correlation coefficient (Q) was used to 
calculate the statistical significance of association between the expression of analyzed genes ( $\Delta \mathrm{Cq}$ values) and various lesions (CRA, malignant polyp CRC N0 and CRC N+). All the statistical analyses were performed using SPSS analytical software version 24 (IBM Corp.), with a cut-off of $\mathrm{P} \leq 0.05$.

\section{Results}

Expression of DCN, EPHA4, FN1, SPARC, SPON2 and SPP1 in CRA and malignant polyp groups compared with healthy mucosa control. The majority of genes tested in the present study were downregulated in CRA compared with healthy mucosa: DCN (16.1-fold, $\mathrm{P}<0.001)$, FN1 (8.1-fold, $\mathrm{P}=0.022$ ), SPARC (2.6-fold, not significant), SPON2 (2.7-fold, not significant) and SPP1 (21.1-fold, $\mathrm{P}=0.006$ ), whereas EPHA4 was slightly upregulated but not statistically significant. Results are summarized in Fig. 1 and Table III.

DCN was significantly downregulated in the malignant polyp group compared with healthy mucosa (4.1-fold, $\mathrm{P}=0.031$; Fig. 1). FN1 and SPP1 also appeared downregulated in malignant polyps compared with healthy mucosa, however these changes were not significant. The other three genes exhibited a trend towards upregulation in malignant polyps, albeit not significant. Results are summarized in Fig. 1 and Table III.

Expression of DCN, EPHA4, FN1, SPARC, SPON2 and SPP1 in CRC N+ and CRC NO groups compared with healthy colon mucosa control. Expression of genes in CRC N0 compared with healthy colon mucosa exhibited a significant upregulation of the levels of SPARC (12.17-fold, P<0.001) and SPON2 (5.65-fold, P<0.001). DCN, EPHA4 and SPP1 expression levels were slightly upregulated and FN1 was downregulated, however these changes were not significant. Results are summarized in Fig. 1 and Table III.

Expression of DCN, EPHA4, SPARC, SPON2 and SPP1 showed similar patterns in the CRC N+ and CRC N0 groups, both compared to their corresponding healthy mucosa. Statistical significance was reached for SPARC (17.3-fold, $\mathrm{P}<0.001)$, SPON2 (12.17-fold, $\mathrm{P}<0.0017)$ and SPP1 (20.65-fold, $\mathrm{P}<0.001)$ expression levels. In contrast to CRC N0, FN1 was upregulated (5.5-fold) in CRC N+ when compared with healthy mucosa, although this change was not statistically significant. Results are summarized in Fig. 1 and Table III.

Expression of DCN, EPHA4, FN1, SPARC, SPON2 and SPPI in CRA compared with the malignant polyp, $C R C N+$ or CRC NO groups. Statistically significant difference between the CRA and malignant polyp groups was observed for the SPON2 and SPARC genes. In addition, statistically significant difference between the CRA and the CRC N0 and CRC N+ groups was observed for all genes tested, except for EPHA4. Results are summarized in Table III.

Expression of most of the genes gradually increased with the level of malignancy, from CRA to malignant polyps and to CRC. Accordingly, the results demonstrated a statistically significant association between the various lesions (CRA, malignant polyp, CRC N0, CRC N+) and the expression levels of DCN, EPHA4, FN1, SPARC, SPON2 and SPP1 $(\varrho=0.660, P<0.001 ; \varrho=-0.259$, $\mathrm{P}=0.039 ; \varrho=0.495, \mathrm{P}<0.001 ; \varrho=0.531, \mathrm{P}<0.001 ; \varrho=0.505$, $\mathrm{P}<0.001$; and $\varrho=0.677, \mathrm{P}<0.001$, respectively). 
Table III. Genes with statistically significant differential expression between two groups.

\begin{tabular}{|c|c|c|c|c|}
\hline Groups & $\begin{array}{c}\text { CRA } \\
(n=10)\end{array}$ & $\begin{array}{l}\text { Malignant polyp } \\
\qquad(\mathrm{n}=10)\end{array}$ & $\begin{array}{l}\text { CRC N0 } \\
(\mathrm{n}=10)\end{array}$ & $\begin{array}{l}\mathrm{CRCN}+ \\
(\mathrm{n}=10)\end{array}$ \\
\hline Healthy mucosa $(n=20)$ & $\begin{array}{l}\mathrm{DCN}(\mathrm{P}<0.001) \\
\text { FN1 }(\mathrm{P}=0.022) \\
\mathrm{SPP} 1(\mathrm{P}=0.006)\end{array}$ & $\mathrm{DCN}(\mathrm{P}=0.031)$ & $\begin{array}{l}\text { SPON2 }(\mathrm{P}<0.001) \\
\text { SPARC }(\mathrm{P}<0.001)\end{array}$ & $\begin{array}{l}\text { SPON2 }(\mathrm{P}<0.001) \\
\text { SPARC }(\mathrm{P}<0.001) \\
\text { SPP1 }(\mathrm{P}<0.001)\end{array}$ \\
\hline $\operatorname{CRA}(n=10)$ & - & $\begin{array}{l}\text { SPON2 }(P=0.028) \\
\text { SPARC }(P=0.018)\end{array}$ & $\begin{array}{l}\text { DCN }(\mathrm{P}<0.001) \\
\text { FN1 }(\mathrm{P}=0.041) \\
\text { SPON2 }(\mathrm{P}<0.001) \\
\text { SPARC }(\mathrm{P}<0.001) \\
\text { SPP1 }(\mathrm{P}<0.001)\end{array}$ & $\begin{array}{l}\text { DCN }(\mathrm{P}<0.001) \\
\text { FN1 }(\mathrm{P}<0.001) \\
\text { SPON2 }(\mathrm{P}<0.001) \\
\text { SPARC }(\mathrm{P}<0.001) \\
\text { SPP1 }(\mathrm{P}<0.001)\end{array}$ \\
\hline
\end{tabular}

CRA, colorectal adenoma; CRC, colorectal carcinoma; N0, no lymph node metastases; $\mathrm{N}+$, with lymph node metastases.

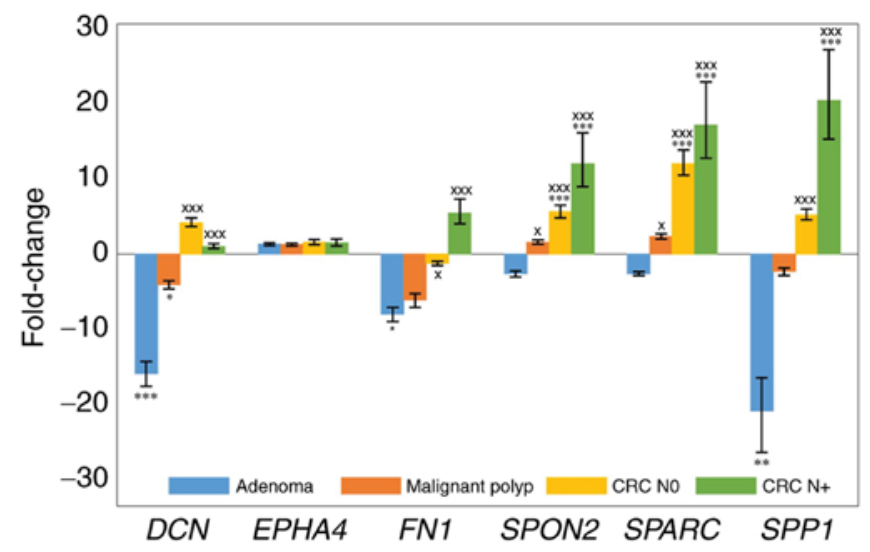

Figure 1. Expression of DCN, EPHA4, FN1, SPARC, SPON2 and SPP1 in adenoma, malignant polyp, CRC N0 and CRC N+ groups. Expression is plotted as fold change relative to healthy colon mucosa tissue control. ${ }^{*} \mathrm{P} \leq 0.05,{ }^{* * *} \mathrm{P}<0.01$ and ${ }^{* * *} \mathrm{P}<0.001$ vs. healthy mucosa; ${ }^{\times} \mathrm{P} \leq 0.05$ and ${ }^{\mathrm{x} \times} \mathrm{P}<0.001$ vs. adenoma. DCN, decorin; EPHA4, erythropoietin-producing hepatoma receptor A4; FN1, fibronectin 1; SPARC, secreted protein acidic and cysteine rich; SPON2, spondin 2; SPP1, secreted phosphoprotein 1; CRC, colorectal carcinoma; N0, no lymph node metastases; N+, with lymph node metastases.

Expression of DCN, EPHA4, FN1, SPARC, SPON2 and $S P P 1$ in CRC N+ compared with CRC NO group. Expression of DCN was slightly upregulated in CRC N0 compared with CRC N+. The difference in DCN expression between CRC N0 and CRC N+ was statistically significant $(\mathrm{P}=0.025)$. There was no significant change in the expression levels of EPHA4, FN1, SPARC, SPON2 and SPP1 between the CRC N0 and $\mathrm{CRC} \mathrm{N}+$ groups. The expression levels of EPHA4 were upregulated compared with healthy mucosa, but the same in CRC NO and CRC N+. FN1 expression was slightly downregulated in CRC NO and upregulated in CRC N+, compared with healthy mucosa. The expression of SPP1, SPON2, and SPARC was upregulated both in CRC N0 and CRC N+ compared with healthy mucosa, and expression of these genes was slightly but not significantly higher in CRC N+than in CRC NO. The P-value of the difference of the SPP1 levels between the CRC N0 and CRC N+ groups was just above the cut-off ( $\mathrm{P}=0.083)$.

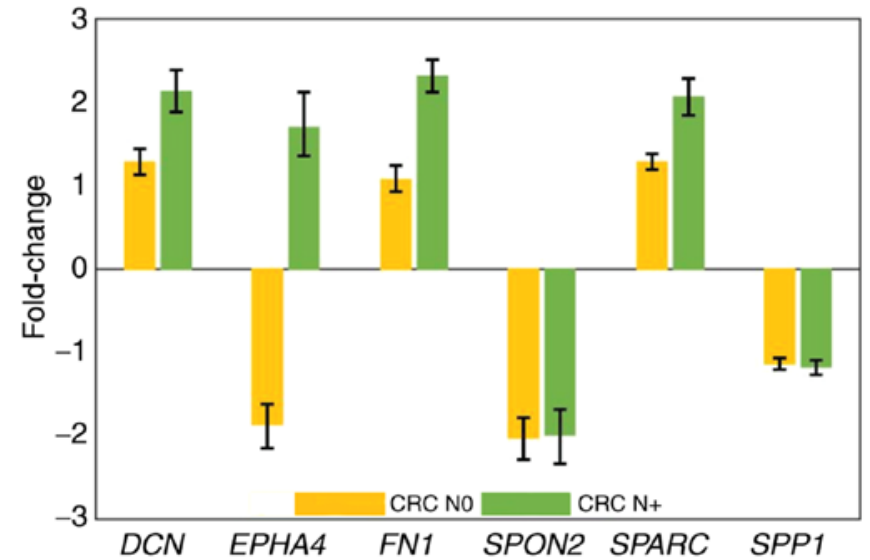

Figure 2. Expression of DCN, EPH4, FN1, SPARC, SPON2 and SPP1 at the invasive front compared with the tumour centre in the CRC NO and $\mathrm{CRC} \mathrm{N}+$ groups. Expression is plotted as fold change relative to the tumor tissue center. DCN, decorin; EPHA4, erythropoietin-producing hepatoma receptor A4; FN1, fibronectin 1; SPARC, secreted protein acidic and cysteine rich; SPON2, spondin 2; SPP1, secreted phosphoprotein 1; CRC, colorectal carcinoma; N0, no lymph node metastases; N+, with lymph node metastases.

Expression of DCN, FN1 and SPARC was upregulated at the invasive front of the tumour compared with the central parts in the CRC N+ group, whereas in the CRC N0 group, expression at the invasive front was similar to the central parts (Fig. 2). The expression levels of SPON2 and SPP1 were downregulated at the invasive front compared with the central parts and similar in both CRC N0 and CRC N+ (Fig. 2). The highest difference in expression levels was observed in the case of the EPHA4 gene; however, there was no statistically significant difference, even though its expression was downregulated in CRC N0 and upregulated in CRC N+ at the invasive front compared with the central parts of the tumour (Fig. 2).

Immunohistochemistry. Expression of most of the genes tested in the present study appeared increased with the level of malignancy, from CRA to malignant polyps and to CRC. Therefore, immunohistochemistry was performed for the DCN, SPARC, SPON2 and SPP1 proteins, since these four genes exhibited statistically significant differences in expression using RT-qPCR between CRC N0 and its corresponding 

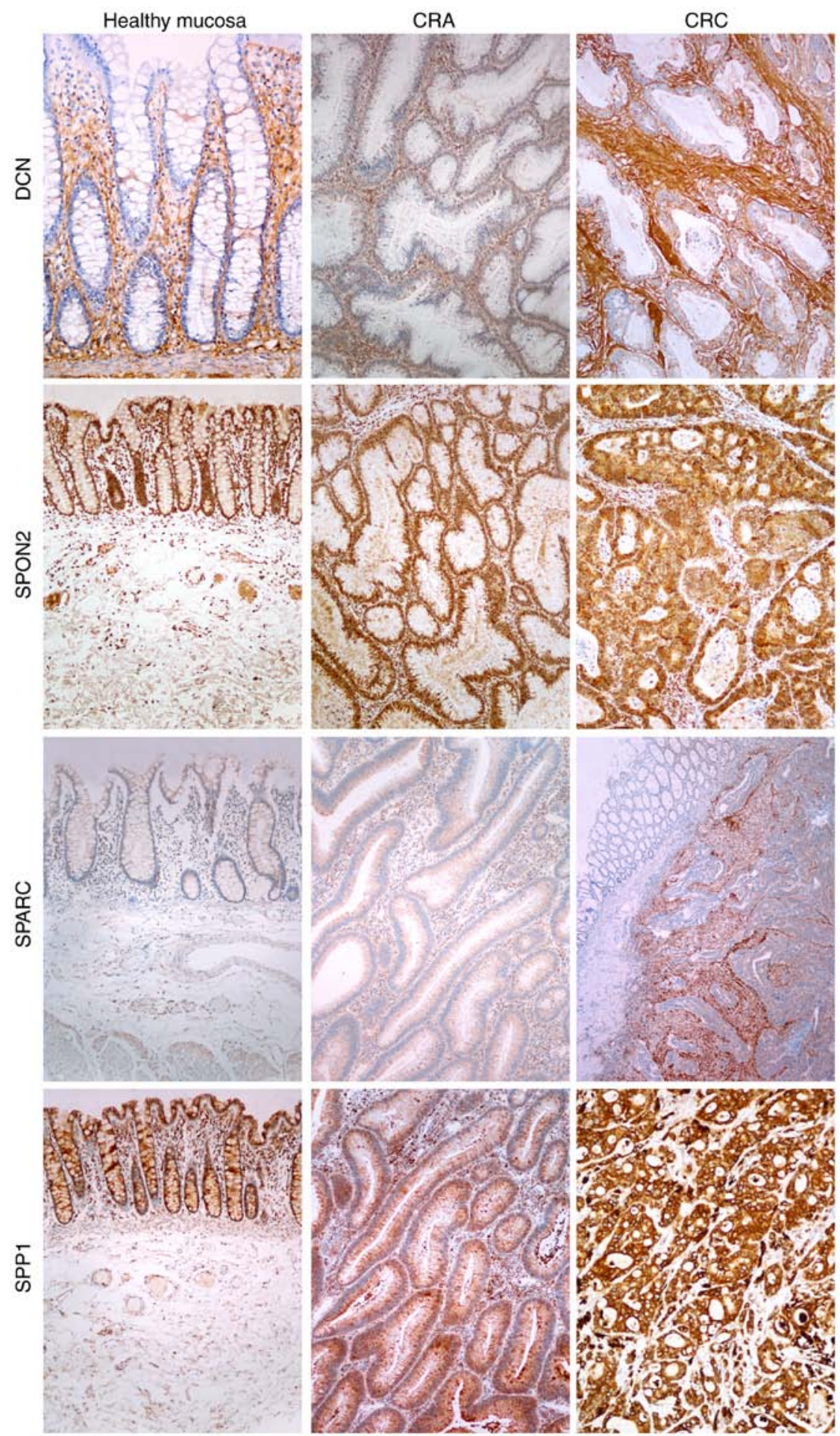

Figure 3. Immunohistochemical analysis of DCN, SPARC, SPP1 and SPON2 expression in healthy colon mucosa, CRA and CRC. SPP1 and SPON2 exhibited positive staining in epithelial cells in healthy mucosa, in dysplastic glands, in CRA and CRC, with the most intense staining being in CRC. DCN staining was positive in the lamina propria in healthy mucosa and in the stroma in CRA and CRC, with the most intense staining being in CRC. SPARC staining was negative in healthy mucosa, very faint in CRA and strong in the stroma in CRC with submucosal invasion. DCN, decorin; SPARC, secreted protein acidic and cysteine rich; SPP1, secreted phosphoprotein 1; SPON2, spondin 2; CRA, colorectal adenoma; CRC, colorectal carcinoma.

healthy mucosa (DCN, 4.22-fold, $\mathrm{P}=0.028$; SPARC, 12.17-fold, $\mathrm{P}=0.007$; SPON2, 5.65-fold, $\mathrm{P}=0.017$; and SPP1, 5.24-fold, $\mathrm{P}=0.036$; Fig. 1).

Immunohistochemistry analysis for the investigated proteins revealed two patterns. One pattern was observed for the SPON2 and SPP1 protein expression: Positive staining was evident in the epithelial cells of the healthy colon mucosa and in dysplastic glands, both in CRA and CRC tissues, and the intensity of staining progressively increased with the severity of the lesions, being the most intense in CRC (Fig. 3).

The other pattern was observed for the DCN and SPARC protein expression: Positive staining was evident in the lamina propria in healthy mucosa and in the stroma of CRA and CRC 
(Fig. 3). In this pattern, too, the intensity of staining increased with the severity of the lesions, being most intense in CRC (Fig. 3).

\section{Discussion}

Based on a previous bioinformatics analysis, six ECM-related genes were identified to be differentially expressed among healthy colon mucosa, CRA and CRC. The present study performed a validation analysis of these six genes and confirmed significant differences in the expression of three genes, namely SPON2, SPARC and SPP1. These were downregulated in healthy mucosa and CRA, and upregulated in CRC. In addition, immunohistochemistry analysis of the related proteins showed patterns that were consistent with the gene expression analysis.

The highest differences in expression among healthy mucosa, CRA and CRC were observed for SPP1: It was downregulated in CRA, but upregulated in $\mathrm{CRC}$, with a gradual increase in its expression from malignant polyp to $\mathrm{CRC}$; the differences among the 3 groups were statistically significant. However, the significance of the difference in expression between $\mathrm{CRC}$ with and $\mathrm{CRC}$ without lymph node metastases was just above the cut-off P-value, and no significant difference was observed between central parts of the tumour and the invasive front. These results suggested an important role of SPP1 in the development of CRC, but not in the progression of CRC. SPP1 is an extracellular protein involved in biomineralization, tissue and bone remodelling, cell-mediated immune response and wound healing $(42,43)$. Previous studies have also suggested that SPP1 has an important role in the development of multiple types of cancer, including CRC, breast, prostate, lung, ovarian and liver cancer (42). For CRC, few studies have been reported on analysis of SPP1 expression using qPCR and immunohistochemistry. However, in contrast to the present findings, upregulation of SPP1 has been reported in CRA and CRC in previous studies $(17,18)$. The present immunohistochemical analysis revealed cytoplasmic expression of SPP1 in epithelial cells in healthy colon mucosa, CRA and CRC, and also in some mesenchymal stromal cells and immune cells. The extent and intensity of staining tended to increase from healthy colon mucosa to CRA and CRC, similarly to previous studies (15-19).

Significant differences were also observed for SPARC: It was slightly downregulated in CRA but upregulated in CRC, with a gradual increase in its expression from malignant polyp to CRC, with statistically significant differences among all three groups. However, there was no significant difference between CRC with and CRC without lymph node metastases. These results indicate a specific role of SPARC in reorganization of ECM in the progression of CRA to CRC. The present results are similar to the results showed by Galamb et al (18), but not quite consistent with another study (35), which showed a slight upregulation in CRA in comparison to healthy tissue, whereas the present results showed a slight downregulation. A previous immunohistochemical analysis (37) reported that SPARC expression was higher in CRC of all stages; however, a statistically significant difference was observed between CRC stage I compared with stages II, III or IV. Liu et al (21) showed that the staining of SPARC was greater in CRA and
CRC without lymph node metastases, whereas the expression of SPARC was still high but to a lesser extent in CRC with lymph node metastases compared to CRC without lymph node metastases. Previously published studies indicate that expression of SPARC in CRC might also be regulated by methylation $(44,45)$, leading to various expression patterns. The SPARC protein, also referred to as osteonectin, is also known to have different expression patterns in various types of cancer, with expression being high in breast cancer, melanoma and glioblastoma, but low in acute myeloid leukaemia, ovarian and pancreatic cancer (46). It also participates in tissue remodelling, wound repair, cell migration and angiogenesis $(46,47)$.

SPON2 exhibited similar expression patterns to those of SPARC. The SPON2 gene encodes for the protein spondin 2, a secretory extracellular protein, that participates in the immune response as a microbial pattern-recognition protein (26). It has been reported that SPON2 is upregulated in several types of cancer, such as lung adenocarcinoma (48), hepatocellular carcinoma (49) and gastric carcinoma (50). A previous study showed that the SPON2 mRNA expression levels are higher in CRC compared with the adjacent healthy mucosa (26). Another study also demonstrated positive SPON2 staining in epithelial cells in CRC (26). The present results are consistent with these previous studies. Furthermore, the present results demonstrated that the expression levels of SPON2 increased with the level of malignancy. Similarly, the differences in expression of SPON2 between the CRC stages examined in the present study were all statistically significant, except between CRC with and CRC without lymph node metastases, indicating that SPON2 may participate in the reorganization of ECM from CRA to CRC

The fourth gene, DCN, encodes for the protein decorin, a member of the small leucine-rich proteoglycans. DCN is a structural component of the ECM, but it also participates in cellular processes, such as proliferation, migration and differentiation (51). The present study demonstrated downregulation of DCN in CRA and malignant polyps but upregulation in CRC, with statistical differences among all groups except between CRA and malignant polyp. Furthermore, among the investigated genes only DCN showed significantly different expression between CRC without lymph node metastases and CRC with lymph node metastases, being higher in CRC without lymph node metastases than in CRC with lymph node metastases. Consistently, DCN has been reported to be negatively correlated with the progression of CRC $(20,51,52)$. With its diverse functions, DCN may have an important role in the development of CRA, progression of CRA to CRC and migration of tumour cells to the regional lymph nodes, forming nodal metastases.

The next gene, FN1, is a widely expressed glycoprotein of the ECM that participates in cell adhesion, migration and differentiation $(23,53)$. It has been demonstrated that FN1 expression is upregulated in CRC (53) and that its immunohistochemical staining is stronger in CRC than in healthy colon mucosa (23); however, no data has been currently available regarding FN1 expression in CRA. In the present study, the results demonstrated downregulation of FN1 in CRA and malignant polyp compared with CRC, regardless of the presence of lymph node metastases, suggesting its role in the development of CRC. There was, however, no difference 
between CRC without lymph node metastases and CRC with lymph node metastases.

Analysis of EPHA4 expression showed no significant differences among the four tissue groups; its expression remained fairly constant during the different stages of CRC development. EPHA4 is a transmembrane receptor tyrosine kinase that is attached to the membrane either by glycosylphosphatidylinositol linkage or a transmembrane sequence (54). Its activation affects pathways involved in the regulation of adhesion, migration and invasiveness (55). Previous studies of EPHA4 in CRC are consistent with the present results. Expression levels of EPHA4 were similar in CRC compared with healthy colon mucosa. There were no significant differences between the expression of EPHA4 in CRC without lymph node metastases and CRC with lymph node metastases (29).

Comparison between the central parts of CRC tissues and the invasive front revealed an upregulation for four out of the six genes at the invasive front, namely for the genes DCN, EPHA4, FN1 and SPARC, particularly in CRC with lymph node metastases. This observation indicates that ECM-related genes may have a role in the progression of CRC.

The present study has several limitations. The first one is related to the fact that the current study was performed on FFPE tissue. Although nucleic acids from FFPE tissues are difficult to analyse, a great advantage of FFPE tissue is that all samples have been first evaluated by pathologists, enabling appropriate diagnosis. RNA fragmentation in FFPE tissue samples has a size endpoint of $\sim 80 \mathrm{nt}$ and formalin modifications are partially reversed during isolation $(56,57)$. Recent publications described that analysis of mRNAs from FFPE is reliable when performed appropriately $(58,59)$. In the present study, only the samples that successfully passed initial quality control were further analysed, thus limiting the number of included samples. The second limitation is related to the different stages of CRC. The number of samples was too small to draw any definite conclusions regarding the correlations between stage and the expression of the investigated genes and proteins. Furthermore, the current study did not investigate the causes of deregulation for these six ECM genes which may be based on DNA, such as methylation, mutation and copy number alteration.

In conclusion, the present study suggested that at least three out of six ECM-related genes, identified by a previous bioinformatics analysis, exhibited significant differences in expression among healthy colon mucosa, CRA and CRC. These results provided further evidence that ECM-related genes and proteins may have an important role in the development of CRC, and identified a possible diagnostic use for these genes in differentiating among various lesions, such as between CRA and malignant polyp. However, the results showed limited potential of these genes to predict lymph node metastases in CRC.

\section{Acknowledgements}

Not applicable.

\section{Funding}

This study was funded by the Slovenian Research Agency (research core funding no. P3-0054).

\section{Availability of data and materials}

The datasets generated and/or analysed during the current study are not publicly available due to protecting confidentiality of personal data but are available from the corresponding author.

\section{Authors' contributions}

MZ contributed to acquisition, analysis and interpretation of data and writing the manuscript. EB contributed to analysis and interpretation of data and in drafting and revising the manuscript. NH contributed to the design of study and critically revised the manuscript. NZ contributed to analysis and interpretation of data, in drafting the manuscript and gave final approval of the version to be published. All authors read and approved the final manuscript.

\section{Ethical approval and consent to participate}

The research project confirmed to the principles outlined in The Helsinki Declaration and was approved by the National Medical Ethics Committee of the Republic of Slovenia, approval no. 0120-88/2018/4. As stated in the approval document, the study is retrospective and observational, performed on tissue samples that were obtained during routine diagnostic/ therapeutic procedures, consisted of either excision or resection. Therefore, enough tissue was available for routine analysis and research. Moreover, tissue is still available for any additional analysis in the future. Our State Ethical Committee does not require informed consent from patients in such studies.

\section{Patient consent for publication}

Not applicable.

\section{Competing interests}

The authors declare that they have no competing interests.

\section{References}

1. Simon K: Colorectal cancer development and advances in screening. Clin Interv Aging 11: 967-976, 2016.

2. Mathonnet M, Perraud A, Christou N, Akil H, Melin C, Battu S, Jauberteau MO and Denizot Y: Hallmarks in colorectal cancer: Angiogenesis and cancer stem-like cells. World J Gastroenterol 20: 4189-4196, 2014.

3. Kuipers EJ, Grady WM, Lieberman D, Seufferlein T, Sung JJ, Boelens PG, van de Velde CJ and Watanabe T: Colorectal cancer. Nat Rev Dis Primers 1: 15065, 2015.

4. Shepherd NA and Griggs RK: Bowel cancer screening-generated diagnostic conundrum of the century: Pseudoinvasion in sigmoid colonic polyps. Mod Pathol 1(Suppl 28): S88-S94, 2015.

5. Griggs RK, Novelli MR, Sanders DS, Warren BF, Williams GT, Quirke P and Shepherd NA: Challenging diagnostic issues in adenomatous polyps with epithelial misplacement in bowel cancer screening: 5 years' experience of the bowel cancer screening programme expert board. Histopathology 70: 466-472, 2017.

6. Panarelli NC, Somarathna T, Samowitz WS, Kornacki S, Sanders SA, Novelli MR, Shepherd NA and Yantiss RK: Diagnostic challenges caused by endoscopic biopsy of colonic polyps: A systematic evaluation of epithelial misplacement with review of problematic polyps from the bowel cancer screening program, United Kingdom. Am J Surg Pathol 40: 1075-1083, 2016. 
7. Loughrey MB and Shepherd NA: The pathology of bowel cancer screening. Histopathology 66: 66-77, 2015.

8. Aghagolzadeh P and Radpour R: New trends in molecular and cellular biomarker discovery for colorectal cancer. World J Gastroenterol 22: 5678-5693, 2016.

9. Hauptman N, Boštjančič E, Žlajpah M, Ranković B and Zidar N: Bioinformatics analysis reveals most prominent gene candidates to distinguish colorectal adenoma from adenocarcinoma. Biomed Res Int 2018: 9416515, 2018.

10. Lu P, Takai K, Weaver VM and Werb Z: Extracellular matrix degradation and remodeling in development and disease. Cold Spring Harb Perspect Biol 3: a005058, 2011.

11. Lu P, Weaver VM and Werb Z: The extracellular matrix: A dynamic niche in cancer progression. J Cell Biol 196: 395-406, 2012.

12. Venning FA, Wullkopf L and Erler JT: Targeting ECM disrupts cancer progression. Front Oncol 5: 224, 2015.

13. Pickup MW, Mouw JK and Weaver VM: The extracellular matrix modulates the hallmarks of cancer. EMBO Rep 15: 1243-1253, 2014.

14. Fang M, Yuan J, Peng C and Li Y: Collagen as a double-edged sword in tumor progression. Tumour Biol 35: 2871-2882, 2014.

15. Boudjadi S, Bernatchez G, Beaulieu JF and Carrier JC: Control of the human osteopontin promoter by ERRalpha in colorectal cancer. Am J Pathol 183: 266-276, 2013.

16. Imano M, Okuno K, Itoh T, Ishimaru E, Satou T and Shiozaki H: Increased osteopontin-positive macrophage expression in colorectal cancer stroma with synchronous liver metastasis. World J Surg 34: 1930-1936, 2010

17. Rohde F, Rimkus C, Friederichs J, Rosenberg R, Marthen C, Doll D, Holzmann B, Siewert JR and Janssen KP: Expression of osteopontin, a target gene of de-regulated Wnt signaling, predicts survival in colon cancer. Int J Cancer 121: 1717-1723, 2007.

18. Galamb O, Sipos F, Spisak S, Galamb B, Krenacs T, Valcz G Tulassay Z and Molnar B: Potential biomarkers of colorectal adenoma-dysplasia-carcinoma progression: mRNA expression profiling and in situ protein detection on TMAs reveal 15 sequentially upregulated and 2 downregulated genes. Cell Oncol 31 19-29, 2009.

19. Valcz G, Sipos F, Krenacs T, Molnar J, Patai AV, Leiszter K, Toth K, Solymosi N, Galamb O, Molnar B and Tulassay Z: Elevated osteopontin expression and proliferative/apoptotic ratio in the colorectal adenoma-dysplasia-carcinoma sequence. Pathol Oncol Res 16: 541-545, 2010

20. Augoff K, Rabczynski J, Tabola R, Czapla L, Ratajczak K and Grabowski K: Immunohistochemical study of decorin expression in polyps and carcinomas of the colon. Med Sci Monit 14: CR530-CR535, 2008

21. Liu QZ, Gao XH, Chang WJ, Wang HT, Wang H, Cao GW and Fu CG: Secreted protein acidic and rich in cysteine expression in human colorectal cancer predicts postoperative prognosis. Eur Rev Med Pharmacol Sci 19: 1803-1811, 2015.

22. Nyman MC, Sainio AO, Pennanen MM, Lund RJ, Vuorikoski S, Sundström JT and Järveläinen HT: Decorin in human colon cancer: Localization in vivo and effect on cancer cell behavior in vitro. J Histochem Cytochem 63: 710-720, 2015.

23. Cai X, Liu C, Zhang TN, Zhu YW, Dong X and Xue P. Down-regulation of FN1 inhibits colorectal carcinogenesis by suppressing proliferation, migration, and invasion. J Cell Biochem 119: 4717-4728, 2018

24. Thorsen SB, Lundberg M, Villablanca A, Christensen SL, Belling KC, Nielsen BS, Knowles M, Gee N, Nielsen HJ, Brunner N, et al: Detection of serological biomarkers by proximity extension assay for detection of colorectal neoplasias in symptomatic individuals. J Transl Med 11: 253 2013.

25. Wang S, Zhang C, Zhang Z, Qian W, Sun Y, Ji B, Zhang Y, Zhu C, Ji D, Wang Q and Sun Y: Transcriptome analysis in primary colorectal cancer tissues from patients with and without liver metastases using next-generation sequencing. Cancer Med 6: 1976-1987, 2017.

26. Zhang Q, Wang XQ, Wang J, Cui SJ, Lou XM, Yan B, Qiao J, Jiang YH, Zhang LJ, Yang PY and Liu F: Upregulation of spondin-2 predicts poor survival of colorectal carcinoma patients. Oncotarget 6: 15095-15110, 2015.

27. Zhao M, Liang F, Zhang B, Yan W and Zhang J: The impact of osteopontin on prognosis and clinicopathology of colorectal cancer patients: A systematic meta-analysis. Sci Rep 5: 12713, 2015 .
28. Sethi MK, Thaysen-Andersen M, Kim H, Park CK, Baker MS, Packer NH, Paik YK, Hancock WS and Fanayan S: Quantitative proteomic analysis of paired colorectal cancer and non-tumorigenic tissues reveals signature proteins and perturbed pathways involved in CRC progression and metastasis. J Proteomics 126 : 54-67, 2015.

29. Oshima T, Akaike M, Yoshihara K, Shiozawa M, Yamamoto N, Sato T, Akihito N, Nagano Y, Fujii S, Kunisaki C, et al: Overexpression of EphA4 gene and reduced expression of EphB2 gene correlates with liver metastasis in colorectal cancer. Int J Oncol 33: 573-577, 2008.

30. Adany R and Iozzo RV: Hypomethylation of the decorin proteoglycan gene in human colon cancer. Biochem J 276: 301-306, 1991

31. Tang RY, Wang Z, Chen HQ and Zhu SB: Negative correlation between miR-200c and decorin plays an important role in the pathogenesis of colorectal carcinoma. Biomed Res Int 2017: 1038984, 2017

32. Liang JF, Wang HK, Xiao H, Li N, Cheng CX, Zhao YZ, Ma YB, Gao JZ, Bai RB and Zheng HX: Relationship and prognostic significance of SPARC and VEGF protein expression in colon cancer. J Exp Clin Cancer Res 29: 71, 2010.

33. Heitzer E, Artl M, Filipits M, Resel M, Graf R, Weissenbacher B, Lax S, Gnant M, Wrba F, Greil R, et al: Differential survival trends of stage II colorectal cancer patients relate to promoter methylation status of PCDH10, SPARC, and UCHL1. Mod Pathol 27: 906-915, 2014

34. Zhang S, Jin J, Tian X and Wu L: hsa-miR-29c-3p regulates biological function of colorectal cancer by targeting SPARC. Oncotarget 8: 104508-104524, 2017.

35. Viana Lde S, Affonso RJ Jr, Silva SR, Denadai MV, Matos D, Salinas de Souza C and Waisberg J: Relationship between the expression of the extracellular matrix genes SPARC, SPP1, FN1, ITGA5 and ITGAV and clinicopathological parameters of tumor progression and colorectal cancer dissemination. Oncology 84: 81-91, 2013.

36. Mlakar V, Berginc G, Volavsek M, Stor Z, Rems M and Glavac D: Presence of activating KRAS mutations correlates significantly with expression of tumour suppressor genes DCN and TPM1 in colorectal cancer. BMC Cancer 9: 282, 2009

37. Chew A, Salama P, Robbshaw A, Klopcic B, Zeps N, Platell C and Lawrance IC: SPARC, FOXP3, CD8 and CD45 correlation with disease recurrence and long-term disease-free survival in colorectal cancer. PLoS One 6: e22047, 2011.

38. Choe EK, Yi JW, Chai YJ and Park KJ: Upregulation of the adipokine genes ADIPOR1 and SPP1 is related to poor survival outcomes in colorectal cancer. J Surg Oncol 117: 1833-1840, 2018.

39. Archer. PreSeq ${ }^{\mathrm{TM}}$ RNA QC Assay. Tecnical note. https://archerdx. com/support/preseq-dna. June 17, 2019.

40. Latham GJ: Normalization of microRNA quantitative RT-PCR data in reduced scale experimental designs. Methods Mol Biol 667: 19-31, 2010.

41. Goni R, Garcia P and Foissac S: The qPCR data statistical analysis. Integromics White Paper 2009. https://gene-quantification.de/integromics-qpcr-statistics-white-paper.pdf. June 17 2019.

42. Wei R, Wong JPC and Kwok HF: Osteopontin-a promising biomarker for cancer therapy. J Cancer 8: 2173-2183, 2017.

43. Shevde LA and Samant RS: Role of osteopontin in the pathophysiology of cancer. Matrix Biol 37: 131-141, 2014.

44. Yang E, Kang HJ, Koh KH, Rhee H, Kim NK and Kim H: Frequent inactivation of SPARC by promoter hypermethylation in colon cancers. Int J Cancer 121: 567-575, 2007.

45. Cheetham S, Tang MJ, Mesak F, Kennecke H, Owen D and Tai IT: SPARC promoter hypermethylation in colorectal cancers can be reversed by 5-Aza-2'deoxycytidine to increase SPARC expression and improve therapy response. Br J Cancer 98: 1810-1819, 2008

46. Tai IT and Tang MJ: SPARC in cancer biology: Its role in cancer progression and potential for therapy. Drug Resist Updat 11: 231-246, 2008

47. Vaz J, Ansari D, Sasor A and Andersson R: SPARC: A potential prognostic and therapeutic target in pancreatic cancer. Pancreas 44: 1024-1035, 2015.

48. Yuan X, Bian T, Liu J, Ke H, Feng J, Zhang Q, Qian L, Li X, Liu Y and Zhang J: Spondin2 is a new prognostic biomarker for lung adenocarcinoma. Oncotarget 8: 59324-59332, 2017.

49. Feng Y, Hu Y, Mao Q, Guo Y, Liu Y, Xue W and Cheng S: Upregulation of Spondin-2 protein expression correlates with poor prognosis in hepatocellular carcinoma. J Int Med Res 47: 569-579, 2019. 
50. Jin C, Lin JR, Ma L, Song Y, Shi YX, Jiang P, Dong Y and Li XS: Elevated spondin-2 expression correlates with progression and prognosis in gastric cancer. Oncotarget 8: 10416-10424, 2017.

51. Jarvinen TA and Prince S: Decorin: A growth factor antagonist for tumor growth inhibition. Biomed Res Int 2015: 654765, 2015.

52. Liu Z, Yang Y, Zhang X, Wang H, Xu W, Wang H, Xiao F, Bai Z, Yao H, Ma X, et al: An oncolytic adenovirus encoding decorin and granulocyte macrophage colony stimulating factor inhibits tumor growth in a colorectal tumor model by targeting pro-tumorigenic dignals and via immune activation. Hum Gene Ther 28: 667-680, 2017.

53. Yi W, Xiao E, Ding R, Luo P and Yang Y: High expression of fibronectin is associated with poor prognosis, cell proliferation and malignancy via the NF- $\mathrm{KB} / \mathrm{p} 53$-apoptosis signaling pathway in colorectal cancer. Oncol Rep 36: 3145-3153, 2016.

54. Kullander K and Klein R: Mechanisms and functions of Eph and ephrin signalling. Nat Rev Mol Cell Biol 3: 475-486, 2002.
55. de Marcondes PG and Morgado-Diaz JA: The role of EphA4 signaling in radiation-induced EMT-like phenotype in colorectal cancer cells. J Cell Biochem 118: 442-445, 2017.

56. Patel PG, Selvarajah S, Guerard KP, Bartlett JMS, Lapointe J, Berman DM, Okello JBA and Park PC: Reliability and performance of commercial RNA and DNA extraction kits for FFPE tissue cores. PLoS One 12: e0179732, 2017.

57. von Ahlfen S, Missel A, Bendrat $\mathrm{K}$ and Schlumpberger $\mathrm{M}$ Determinants of RNA quality from FFPE samples. PLoS One 2: e1261, 2007.

58. Guerra JM,Monteiro RL, GonzalezL,Kimura LM, Cirqueira CDS and de Araujo LJT: Against all odds: RNA extraction from different protocols adapted to formalin-fixed paraffin-embedded tissue. Appl Immunohistochem Mol Morphol: May 23, 2019 (Epub ahead of print). doi: 10.1097/PAI.0000000000000772.

59. Bustin SA, Benes V, Garson JA, Hellemans J, Huggett J, Kubista M, Mueller R, Nolan T, Pfaffl MW, Shipley GL, et al: The MIQE guidelines: Minimum information for publication of quantitative real-time PCR experiments. Clin Chem 55: 611-622, 2009. 